\title{
THE QUASITOPOS OF B-UNIFORM FILTER SPACES
}

\author{
DIETER LESEBERG AND ZOHREH VAZIRY
}

\begin{abstract}
In this paper a systematic study begins of the category b-UFIL of buniform filter spaces and b-uniformly continuous mappings. We will show that this construct forms a quasitopos in which quotients are stable under products. Consequently, it is extremely useful for further studies in the realm of Bounded topology.
\end{abstract}

\section{INTRODUCTION}

This paper continues our treatise on "Categories of several convergences". The present terminology is essentially the one used in the above mentioned paper [10]. Our focus is the consideration of so-called b-uniform filter spaces, which represent a natural generalization of several classical convergences such as uniform convergences, point-convergences, filtermerotopies and Cauchy spaces or suited set-convergences as well, see $[1,2,4-6,11,13]$. Moreover, following the idea of establishing a more convenient category with well-behaved properties such as being a quasitopos in the sense of Penon or Preuß [11], respectively, we will show that the category b-UFIL of b-uniform filter spaces possesses such desirable features. In addition, we can state that even quotient maps in b-UFIL are closed under formation of arbitrary products. Thus, this new established concept makes it easier for topologists to solve their problems since strong topological universes are extremely useful [11].

On the other hand, b-UFIL can be nicely embedded into the fundamental construct b-CONV of b-convergence spaces and corresponding maps [7-9], which additionally contains all set-convergences and STOP, the category of supertopological spaces and continuous maps in the sense of Doitchinov [3]. Hence, b-UFIL represents a bridge between all classical convergences and the broader concept of b-convergence in the setting of the fundamental concept called Bounded topology, in which structures on bounded sets or bornologies are defined. Here we should note that classical concepts are essentially working on the power set of a given set $X$.

$M S C$ (2010): primary 54A20, 54B30; secondary 54E15.

Keywords: set-convergence, point-convergence, b-uniform filter space, b-filter space, completeness, compactness, precompactness, topological construct, strong topological universe, bounded topology. 


\section{The COnstruct B-UFIL}

Definition 2.1. For a set $X$ a pair $\left(\mathcal{B}^{X}, \mu\right)$ consisting of a non-empty subset $\mathcal{B}^{X} \subset \underline{P} X$ and a non-empty set $\mu \subset F I L(X \times X)$ of uniform filters is called a $b$-uniform filter structure on $X$, and the triple $\left(X, \mathcal{B}^{X}, \mu\right)$ a b-uniform filter space provided that the following axioms are satisfied:

(buf b $_{1} B_{1} \subset B \in \mathcal{B}^{X}$ imply $B_{1} \in \mathcal{B}^{X}$;

(buf $_{2}$ ) $x \in X$ implies $\{x\} \in \mathcal{B}^{X}$;

(buf $_{3}$ ) $B \in \mathcal{B}^{X} \backslash\{\emptyset\}$ implies $\dot{B} \times \dot{B} \in \mu$;

(buf $)_{4} \mathcal{U} \in \mu$ and $\mathcal{U} \subset \mathcal{U}_{1} \in F I L(X \times X)$ imply $\mathcal{U}_{1} \in \mu$.

Here, $\underline{P} X$ denotes the power set of $X, F I L(X \times X)$ is the set of all filters on $X \times X$ and its elements are called uniform filters (on $X$ ) and for $B \in \mathcal{B}^{X} \backslash\{\emptyset\}, \dot{B}:=\{A \subset$ $X: A \supset B\}$.

Given a pair of b-uniform filter spaces $\left(X, \mathcal{B}^{X}, \mu_{X}\right),\left(Y, \mathcal{B}^{Y}, \mu_{Y}\right)$, a map $f$ : $X \longrightarrow Y$ is called b-uniformly continuous, in short buc, if $f$ satisfies the following conditions:

(buc 1 ) $B \in \mathcal{B}^{X}$ implies $f[B] \in \mathcal{B}^{Y}$;

$\left(\right.$ buc $\left._{2}\right) \mathcal{U} \in \mu$ implies $(f \times f)(\mathcal{U}) \in \mu_{Y}$, where $(f \times f)(\mathcal{U}):=\{V \subset Y \times Y$ : $\exists U \in \mathcal{U}$ such that $(f \times f)[U] \subset V\}$ with $(f \times f)[U]:=\left\{(f \times f)\left(x_{1}, x_{2}\right)\right.$ : $\left.\left(x_{1}, x_{2}\right) \in U\right\}=\left\{\left(f\left(x_{1}\right), f\left(x_{2}\right)\right):\left(x_{1}, x_{2}\right) \in U\right\}$.

By b-UFIL we will denote the category of b-uniform filter spaces and b-uniformly continuous maps.

Proposition 2.2. b-UFIL is a topological construct in the sense of [11].

Proof. For a set $X$, let $I$ be a class, $\left(X_{i}, \mu_{i}\right)_{i \in I}$ a family of b-uniform filter spaces and $\left(f_{i}: X \longrightarrow X_{i}\right)_{i \in I}$ a family of maps. Then, $\left(\mathcal{B}_{I}^{X}, \mu_{X}^{I}\right)$ is the initial b-UFIL structure on $X$, where $\mathcal{B}_{I}^{X}:=\left\{B \subset X: \forall i \in I, f_{i}[B] \in \mathcal{B}^{X_{i}}\right\}$ and $\mu_{X}^{I}:=\left\{\mathcal{U} \in F I L(X \times X): \forall i \in I,\left(f_{i} \times f_{i}\right)(\mathcal{U}) \in \mu_{i}\right\}$.

Evidently, $\left(\mathcal{B}_{I}^{X}, \mu_{X}^{I}\right)$ satisfies the axioms $\left(\right.$ buf $\left._{1}\right),\left(\right.$ buf $\left._{2}\right)$, and $\left(\right.$ buf $\left._{4}\right)$, respectively.

To $\left(\right.$ buf $\left._{3}\right)$ : For $B \in \mathcal{B}^{X} \backslash\{\emptyset\}$ we are getting $\left(f_{i} \times f_{i}\right)(\dot{B} \times \dot{B})=f_{i}(\dot{B}) \times f_{i}(\dot{B})$ $=f[\dot{B}] \times f_{i}[\dot{B}] \in \mu_{i}$ for each $i \in I$, hence, $\dot{B} \times \dot{B} \in \mu_{X}^{I}$ follows.

Here, in general, for $\mathcal{U}_{1}, \mathcal{U}_{2} \in F I L(X \times X)$, their cross-product is defined by setting:

$$
\mathcal{U}_{1} \times \mathcal{U}_{2}:=\left\{R \subset X \times X: \exists U_{1} \in \mathcal{U}_{1}, \exists U_{2} \in \mathcal{U}_{2} \text { such that } R \supset U_{1} \times U_{2}\right\} .
$$

By definition of $\left(\mathcal{B}_{I}^{X}, \mu_{X}^{I}\right)$, each $f_{i}$ is b-uniformly continuous. Now let

$$
\left(Y, \mathcal{B}^{Y}, \mu_{Y}\right)
$$

be a b-uniform filter space and $g: Y \longrightarrow X$ be a mapping such that, for each $i \in I, f_{i} \circ g:\left(Y, \mathcal{B}^{Y}, \mu_{Y}\right) \longrightarrow\left(X_{i}, \mathcal{B}^{X_{i}}, \mu_{i}\right)$ is b-uniformly continuous. We have to show that $g:\left(Y, \mathcal{B}^{Y}, \mu_{Y}\right) \longrightarrow\left(X, \mathcal{B}_{I}^{X}, \mu_{X}^{I}\right)$ is b-uniformly continuous.

To $\left(\right.$ buc $\left._{1}\right): B \in \mathcal{B}^{Y}$ implies $\left(f_{i} \circ g\right)[B]=f_{i}[g[B]] \in \mathcal{B}^{X_{i}}$ for each $i \in I$ by the assumption. Hence, $g[B] \in \mathcal{B}_{I}^{X}$ follows.

To $\left(\operatorname{buc}_{2}\right): \mathcal{U} \in \mu_{Y}$ implies $\left(f_{i} \times f_{i}\right)((g \times g)(\mathcal{U}))=\left(\left(f_{i} \circ g\right) \times\left(f_{i} \circ g\right)\right)(\mathcal{U}) \in \mu_{i}$ for each $i \in I$ by the hypothesis. Consequently, $(g \times g)(\mathcal{U}) \in \mu_{X}^{I}$ results, showing 
that $g:\left(Y, \mathcal{B}^{Y}, \mu_{Y}\right) \longrightarrow\left(X, \mathcal{B}_{I}^{X}, \mu_{X}^{I}\right)$ is b-uniformly continuous. Since $\left(\mathcal{B}^{X}, \mu\right) \in$ $\underline{P}(\underline{P} X) \times \underline{P}(F I L(X \times X))$ is valid, the class of all b-uniform filter structures on $X$ is a set.

Finally, the only b-uniform filter structure on a set $X$ with $\operatorname{card} X=1$ is the pair $(\{\emptyset,\{x\}\},\{\dot{x} \times \dot{x}, \underline{P}(\{x\} \times\{x\})\})$, where $x$ denotes the element of $X$. If $X$ is empty, then $(\{\emptyset\},\{\{\emptyset\}\})$ represents the only b-uniform filter structure on $X$.

Remark 2.3. As already observed in our former paper [10], preuniform convergence spaces and b-uniform filter spaces essentially coincide if and only if the assumed boundedness $\mathcal{B}^{X}$ is discrete, meaning that $\mathcal{B}^{X}=\{\emptyset\} \cup\{\{x\}: x \in X\}:=\mathcal{D}^{X}$ (compare with Definition 2.12). Further, we repeat that the corresponding named category DISb-UFIL is bicoreflective in b-UFIL and itself forms a strong topological universe, see [11]. On the other hand, some important set-convergences are in a one to one correspondence to b-uniform filter spaces and, finally, generalized filter merotopies introduced as b-filter spaces can also be nicely embedded into b-UFIL.

Thus, our introduced concept can be regarded as a suitable tool for studying all the mentioned constructs in common. Moreover, in this context let us still mention the fact that basic properties of spaces such as compactness, precompactness and completeness can be newly defined in b-UFIL, and fundamental theorems, as for example that of Tychonoff, find corresponding expressions [10].

Remark 2.4. Since b-UFIL is a topological construct, the set of all b-uniform filter structures on $X$ forms a complete lattice. So the following definition makes sense.

Definition 2.5. Let $X$ be a set and let $\left(\mathcal{B}_{1}^{X}, \mu_{1}\right),\left(\mathcal{B}_{2}^{X}, \mu_{2}\right)$ be b-uniform filter structures on $X$. Then we are setting:

$$
\left(\mathcal{B}_{1}^{X}, \mu_{1}\right) \leq\left(\mathcal{B}_{2}^{X}, \mu_{2}\right) \text { if and only if } \mathcal{B}_{1}^{X} \subset \mathcal{B}_{2}^{X} \text { and } \mu_{1} \subset \mu_{2} \text {. }
$$

$\left(\mathcal{B}_{1}^{X}, \mu_{1}\right)$ is said to be finer than $\left(\mathcal{B}_{2}^{X}, \mu_{2}\right)$ and $\left(\mathcal{B}_{2}^{X}, \mu_{2}\right)$ is said to be coarser than $\left(\mathcal{B}_{1}^{X}, \mu_{1}\right)$.

Remark 2.6. The initial b-uniform filter structure on a set $X$ with respect to $\left(X, f_{i},\left(X_{i}, \mathcal{B}_{i}^{X}, \mu_{i}\right), I\right)$ is the coarsest b-uniform filter structure on $X$ such that $f_{i}$ is b-uniformly continuous for each $i \in I$.

In the event of $I$ being the empty class, $\left(\mathcal{B}_{I}^{X}, \mu_{X}^{I}\right)=(\underline{P} X, F I L(X \times X))$, and it is called an indiscrete b-uniform filter structure on $X$.

Proposition 2.7. Let $\left(X, \mathcal{B}^{X}, \mu\right)$ be a b-uniform filter space and $A \subset X$. Then, $\left(\mathcal{B}^{A}, \mu_{A}\right)$ is a b-uniform filter structure on $A$, where $\mathcal{B}^{A}:=\{B \cap A$ : $\left.B \in \mathcal{B}^{X}\right\}$ and $\mu_{A}:=\left\{\mathcal{U}_{A}: \mathcal{U} \in \mu\right\}$ with $\mathcal{U}_{A}:=\{U \cap A \times A: U \in \mathcal{U}\}$, such that $\left(A, \mathcal{B}^{A}, \mu_{A}\right)$ represents the b-uniform filter subspace of $\left(X, \mathcal{B}^{X}, \mu\right)$ in $\mathbf{b}$-UFIL, meaning that $\left(\mathcal{B}^{A}, \mu_{A}\right)$ is the initial b-uniform filter structure on $A$ with respect to $\left(X, i,\left(X, \mathcal{B}^{X}, \mu\right)\right)$, where $i: A \longrightarrow X$ denotes the inclusion map.

Proof. First let us denote by $i: A \longrightarrow X$ the corresponding inclusion map. Evidently, $\mathcal{B}^{A}$ is not empty.

To (buf but $_{1}$ Let $B_{1} \subset B \cap A$ for some $B \in \mathcal{B}^{X}$. Then, $B_{1} \in \mathcal{B}^{X}$ with $B_{1}=B_{1} \cap A$, and $B_{1} \in \mathcal{B}^{A}$ follows. 
To (buf 2$): x \in A$ implies $\{x\} \in \mathcal{B}^{X}$ with $\{x\}=\{x\} \cap A$, and $\{x\} \in \mathcal{B}^{A}$ results. $\mu_{A}$ is not empty, since $\mu \neq \emptyset$.

First, we show $\mathcal{U}_{A} \in F I L(A \times A)$. $\mathcal{U}_{A} \neq \emptyset$, since $\mathcal{U} \neq \emptyset$. Now let $U_{1} \cap A \times A$ for some $U_{1} \in \mathcal{U}$ and $U_{2} \cap A \times A$ for some $U_{2} \in \mathcal{U}$ be given. Then, $\left(U_{1} \cap A \times A\right) \cap$ $\left(U_{2} \cap A \times A\right)=\left(U_{1} \cap U_{2}\right) \cap(A \times A)$ with $U_{1} \cap U_{2} \in \mathcal{U}$, so that the intersection of the given elements of $\mathcal{U}_{A}$ belongs to $\mathcal{U}_{A}$.

Finally, let $\left(U_{1} \cap A \times A\right) \cap\left(U_{2} \cap A \times A\right)$ be an element of $\mathcal{U}_{A}$ with $U_{1}, U_{2} \in \mathcal{U}$. We have to show that $U_{1} \cap A \times A$ as well as $U_{2} \cap A \times A$ belongs to $\mathcal{U}_{A}$. By the hypothesis, we are getting $\left(U_{1} \cap A \times A\right) \cap\left(U_{2} \cap A \times A\right)=U \cap A \times A$ for some $U \in \mathcal{U}$, hence, $U \cup U_{1} \in \mathcal{U}$ and $U \cup U_{2} \in \mathcal{U}$ follows. But then, $U_{1} \cap A \times A=\left(U \cup U_{1}\right) \cap A \times A$ and $U_{2} \cap A \times A=\left(U \cup U_{2}\right) \cap A \times A$ can be easily deduced showing the claim.

To $\left(\right.$ buf $\left._{3}\right)$ : For $D \in \mathcal{B}^{A} \backslash\{\emptyset\}$, we have to verify that $\dot{D} \times \dot{D} \in \mu_{A}$ holds. By the definition of $\mathcal{B}^{A}$, we are getting $D=B \cap A$ for some $B \in \mathcal{B}^{X}$, hence, $\dot{B} \times \dot{B} \in \mu$ follows, and $\dot{D} \times \dot{D}=\{U \cap A \times A: U \in \dot{B} \times \dot{B}\}=(\dot{B} \times \dot{B})_{A} \in \mu_{A}$ results.

To " $\leq$ ": $R \in \dot{D} \times \dot{D}$ implies $R \supset D \times D$, hence, $R \supset(B \cap A) \times(B \cap A)=$ $(B \times B) \cap(A \times A)=: R_{1}$. Consequently, $R_{1} \in(\dot{B} \times \dot{B})_{A}$ is true, and $R \in(\dot{B} \times \dot{B})_{A}$ follows.

To " $\geq$ ": Conversely, let $R \in(\dot{B} \times \dot{B})_{A}$. Then $R=U \cap A \times A$ for some $U \in \dot{B} \times \dot{B}$, hence, $R \supset(B \times B) \cap(A \times A)=(B \cap A) \times(B \cap A)=D \times D$, and $R \in \dot{D} \times \dot{D}$ is valid.

To $\left(\right.$ buf $\left._{4}\right):$ Now $\mathcal{V} \in F I L(A \times A)$ with $\mathcal{V} \supset \mathcal{U}_{A}$ for some $\mathcal{U} \in \mu$ are implying $(i \times i)(\mathcal{V}) \supset \mathcal{U}$ since $R \in \mathcal{U}$ implies $R \cap A \times A \in \mathcal{U}_{A}$, hence, $R \cap A \times A \in \mathcal{V}$ follows by the hypothesis, and $R \in(i \times i)(\mathcal{V})$ results. Consequently, $(i \times i)(\mathcal{V}) \in \mu$ follows. But $\mathcal{V}=((i \times i)(\mathcal{V}))_{A}$ because $R \in \mathcal{V}$ implies $R=A \times A \cap(i \times i)[R]$, hence, $R \in((i \times i)(\mathcal{V}))_{A}$ is true.

Conversely, $R \in((i \times i)(\mathcal{V}))_{A}$ implies $R=S \cap A \times A$ for some $S \in((i \times i)(\mathcal{V})$. Consequently, we can find $V \in \mathcal{V}$ such that $S \supset(i \times i)[V]=V$ holds. But then, $S \cap A \times A \supset V$, and $R \in \mathcal{V}$ follows. Evidently, $\left\{i[D]: D \in \mathcal{B}^{A}\right\}=: i \mathcal{B}^{A} \subset \mathcal{B}^{X}$ holds, so that $i:\left(A, \mathcal{B}^{A}, \mu_{A}\right) \longrightarrow\left(X, \mathcal{B}^{X}, \mu\right)$ satisfies $\left(\right.$ buc $\left._{1}\right)$.

To $\left(\right.$ buc $\left._{2}\right)$ : Now let $\mathcal{U}_{A} \in \mu_{A}$ for some $\mathcal{U} \in \mu$. We will show that $\mathcal{U} \subset(i \times i)\left(\mathcal{U}_{A}\right)$ can be deduced. $U \in \mathcal{U}$ implies $U \cap A \times A \in \mathcal{U}_{A}$, hence, $U \cap A \times A=(i \times i)[U \cap$ $A \times A] \in(i \times i)\left(\mathcal{U}_{A}\right)$ is valid, and $U \in(i \times i)\left(\mathcal{U}_{A}\right)$ results. Finally, let $\left(\mathcal{E}^{A}, \eta\right)$ be b-uniform filter structure on $A$ with $i:\left(A, \mathcal{E}^{A}, \eta\right) \longrightarrow\left(X, \mathcal{B}^{X}, \mu\right)$ is buc. Our goal is to verify $\left(\mathcal{E}^{A}, \eta\right) \leq\left(\mathcal{B}^{A}, \mu_{A}\right)$. First, let $D \in \mathcal{E}^{A}$. Then, by the hypothesis, $i[D] \in \mathcal{B}^{X}$ is true. But $D=i[D]=D \cap A$, and $D \in \mathcal{B}^{A}$ results.

Next, let $\mathcal{V} \in \eta$, we will show that $\mathcal{V}=\mathcal{U}_{A}$ for some $\mathcal{U} \in \mu$. By the assumption, we know that $(i \times i)(\mathcal{V}) \in \mu$ is true, which means that, by the former proof, $\mathcal{V}=((i \times i)(\mathcal{V}))_{A}$ results. This statement concludes the proof.

Remark 2.8. Since b-UFIL forms a topological construct, there exist arbitrary final structures. The final b-uniform filter structures on a set $X$ with respect to $\left(\left(X_{i}, \mathcal{B}^{X_{i}}, \mu_{i}\right), f_{i}, X, I\right)$, where $f_{i}: X_{i} \longrightarrow X$ are mappings for each $i \in I$, denoted by $\left(\mathcal{B}_{X}^{I}, \mu_{I}^{X}\right)$ is the finest b-uniform filter structure on $X$ such that $f_{i}$ 
is b-uniformly continuous for each $i \in I$, (compare with Definition 2.5 and Remark 2.6, respectively). If $I$ is the empty class, then $\left(\mathcal{B}_{X}^{I}, \mu_{I}^{X}\right)=\left(\mathcal{D}^{X},\{\mathcal{V} \in\right.$ $F I L(X \times X): \exists x \in X$ such that $\dot{x} \times \dot{x} \subset \mathcal{V}\} \cup\{\underline{P}(X \times X)\})$.

Remark 2.9. In general, let $\underline{C}$ be a category. Then a family $\left(f_{i}: X_{i} \longrightarrow X\right)_{i \in I}$

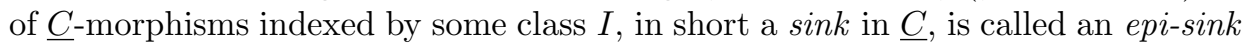
in $\underline{C}$ provided that, for any pair $X \stackrel{\alpha}{\rightarrow} Y$ and $X \stackrel{\beta}{\rightarrow} Y$ of $\underline{C}$-morphisms such that $\alpha \circ f_{i}=\beta \circ f_{i}$ for each $i \in I$, it follows that $\alpha=\beta$.

If $\underline{C}$ is a topological construct with structured sets $(X, \mu)$ as objects, then $f_{i}:\left(X_{i}, \mu_{i}\right) \longrightarrow(X, \mu)$ is called final provided that $\mu$ is the final $\underline{C}$-structure on $X$ with respect to the given data.

Motivation 2.10. In a topological construct $\underline{C}$, final epi-sinks play an important role. So it is possible to describe the extensionality of $\underline{C}$ ( meaning that each $\underline{C}$-object has a one-point extension in $\underline{C}$ ) by the fact that in $\underline{C}$ final epi-sinks are hereditary. Moreover, a construct is Cartesian closed, meaning that in $\underline{C}$ natural function space structures are available, if and only if for each $\underline{C}$-object $\bar{Y}$ and for any final epi-sink $\left(f_{i}: X_{i} \longrightarrow X\right)_{i \in I}$ in $\underline{C}\left(1_{Y} \times f_{i}: Y \times X_{i} \longrightarrow Y \times X\right)_{i \in I}$ is a final epi-sink, i.e., the functor " $Y \times$-" preserves final epi-sinks, see [11].

Proposition 2.11. Let $X$ be a set, $\left(X, \mathcal{B}^{X_{i}}, \mu_{i}\right)_{i \in I}$ a family of b-uniform filter spaces and $\left(f_{i}: X_{i} \longrightarrow X\right)_{i \in I}$ a family of maps. If $\left(f_{i}: X_{i} \longrightarrow X\right)_{i \in I}$ is an epi-sink in $b$-UFIL, then $\left(\mathcal{B}_{X}^{I}, \mu_{I}^{X}\right)$ is the final b-uniform filter structure on $X$ with respect to the given data, where $\mathcal{B}_{X}^{I}:=\left\{B \subset X: \exists i \in I \exists B_{i} \in \mathcal{B}^{X_{i}}\right.$ such that $\left.B \subset f_{i}\left[B_{i}\right]\right\}$ and $\mu_{I}^{X}:=\left\{\mathcal{U} \in F I L(X \times X): \exists i \in I \exists \mathcal{U}_{i} \in \mu_{i}\right.$ such that $\left.\left(f_{i} \times f_{i}\right)\left(\mathcal{U}_{i}\right) \subset \mathcal{U}\right\}$.

Proof. It suffices to show that the equation $X=\bigcup_{i \in I} f_{i}\left[X_{i}\right]$ holds, then the remainder is clear. If card $X<2$, then the assertion is trivial. Let card $X \geq 2$. If $X \neq \bigcup_{i \in I} f_{i}\left[X_{i}\right]$, then there would be $x_{0} \in \bigcup_{i \in I} f_{i}\left[X_{i}\right]$ and $x_{1} \in X \backslash \bigcup_{i \in I} f_{i}\left[X_{i}\right]$. If $\left\{x_{0}, x_{1}\right\}$ is endowed with the indiscrete b-uniform filter structure, see Remark 2.6, then one obtains an object in b-UFIL. Hence, $\alpha: X \longrightarrow Z$ defined by $\alpha(x):=x_{0}$ for each $x \in X$ and $\beta: X \longrightarrow Z$ defined by

$$
\beta(x):= \begin{cases}x_{0} & \text { for } x \in \cup_{i \in I} f_{i}\left[X_{i}\right] \\ x_{1} & \text { otherwise }\end{cases}
$$

are b-uniformly continuous maps such that $\alpha \circ f_{i}=\beta \circ f_{i}$ for each $i \in I$. Obviously, $\alpha \neq \beta$ in contradiction to the fact that $\left(f_{i}\right)_{i \in I}$ is an epi-sink. Consequently, $X=\bigcup_{i \in I} f_{i}\left[X_{i}\right]$ follows.

Next, we will demonstrate how b-UFIL can be nicely embedded into b-CONV, the topological construct of b-convergence spaces and b-continuous maps, see [7] and the following definition. This superconstruct contains not only the neighborhood spaces of Tozzi and Wyler [12] or the supertopological spaces in the sense of Doitchinov [3] but also the set-convergences as defined by Wyler [13]. Hence, convergences in all their facets are now being involved and can be examined for their prevailing aspects.

Definition 2.12. A triple $\left(X, \mathcal{B}^{X}, \tau\right)$ consisting of a set $X$, a boundedness $\mathcal{B}^{X}$ and a convergence function $\tau: \mathcal{B}^{X} \longrightarrow \underline{P}(F I L(X \times X))$ is called b-convergence space provided that $\tau$ satisfies the below conditions: 
$\left(\mathrm{bc}_{1}\right) B \in \mathcal{B}^{X}, \mathcal{U} \in \tau(B)$ and $\mathcal{U} \subset \mathcal{V} \in F I L(X \times X)$ imply $\mathcal{V} \in \tau(B)$;

$\left(\mathrm{bc}_{2}\right) \tau(\emptyset)=\{\underline{P}(X \times X)\}$;

(bc 3$) x \in X$ implies $\dot{x} \times \dot{x} \in \tau(\{x\})$.

Here, a boundedness $\mathcal{B}^{X}$ is a non-empty subset of $\underline{P} X$ that satisfies the following conditions:

(b $\left.\mathrm{b}_{1}\right) B_{1} \subset B \in \mathcal{B}^{X}$ implies $B_{1} \in \mathcal{B}^{X}$;

(b $\left.\mathrm{b}_{2}\right) x \in X$ implies $\{x\} \in \mathcal{B}^{X}$.

Given two b-convergence spaces $\left(X, \mathcal{B}^{X}, \tau_{X}\right),\left(Y, \mathcal{B}^{Y}, \tau_{Y}\right)$ a function $f: X \longrightarrow Y$ is called b-continuous if it is bounded, which means $\left\{f[B]: B \in \mathcal{B}^{X}\right\} \subset \mathcal{B}^{Y}$ and, in addition, we have that $f$ preserves uniform filters in the sense that $B \in \mathcal{B}^{X}$ and $\mathcal{U} \in \tau_{X}(B)$ imply $(f \times f)(\mathcal{U}) \in \tau_{Y}(f[B])$.

Moreover, by b-CONV we will denote the corresponding category.

Remark 2.13. There exist some interesting subcategories of $\mathbf{b}-\mathbf{C O N V}$ that can be described as follows. Let us call a b-convergence space $\left(X, \mathcal{B}^{X}, \tau\right)$

(i) equiform if $\tau$ satisfies the condition

(e) $B_{1}, B_{2} \in \mathcal{B}^{X} \backslash\{\emptyset\}$ imply $\tau\left(B_{1}\right)=\tau\left(B_{2}\right)$;

(ii) set-pointed if $\tau$ satisfies the condition

(sp) $B \in \mathcal{B}^{X}$ implies $\dot{B} \times \dot{B} \in \tau(B)$.

For both definitions, there exist special fundamental convergences independent of each other as presented in [10]. Now, let us call a b-convergence space $\left(X, \mathcal{B}^{X}, \tau\right)$ set-pointed equiform provided $\tau$ satisfies both the above conditions. By SETebCONV we will denote the full subcategory of b-CONV whose objects are the set-pointed equiform b-convergence spaces.

Theorem 2.14. The categories b-UFIL and SETeb-CONV are isomorphic.

Proof. Let $\left(X, \mathcal{B}^{X}, \mu\right)$ be a b-uniform filter space. Then define a convergence function $\tau_{\mu}$ by setting:

$$
\begin{aligned}
& \tau_{\mu}(\emptyset):=\{\underline{P}(X \times X)\} \text { and } \\
& \tau_{\mu}(B):=\mu \text { for each } B \in \mathcal{B}^{X} \backslash\{\emptyset\} .
\end{aligned}
$$

Evidently, $\tau_{\mu}$ satisfies all axioms $\left(\mathrm{bc}_{1}\right)$ To $\left(\mathrm{bc}_{3}\right),(\mathrm{sp})$ and $(\mathrm{e})$, respectively. Conversely, if assuming a set-pointed equiform b-convergence space $\left(Y, \mathcal{B}^{Y}, t\right)$, we put:

$$
\eta_{t}:=\left\{\mathcal{U} \in F I L(Y \times Y): \exists B \in \mathcal{B}^{Y} \text { such that } \mathcal{U} \in t(B)\right\}
$$

Hence, $\left(Y, \mathcal{B}^{Y}, \eta_{t}\right)$ defines a b-uniform filter space so that the following equations hold:

(i) $\eta_{\tau_{\mu}}=\mu$;

(ii) $\tau_{\eta_{t}}=t$.

To (i) " $\geq$ ": Let $X=\emptyset$ and $\mathcal{U} \in \mu$, hence, $\mathcal{U}=\underline{P}(X \times X)=\{\emptyset\}$, according to Proposition 2.2 consequently, $\mathcal{U} \in \tau(\emptyset)$ follows, and $\mathcal{U} \in \eta_{\tau_{\mu}}$ results.

" $\leq$ " Conversely, $\mathcal{U} \in \eta_{\tau_{\mu}}$ implies $\mathcal{U} \in \tau_{\mu}(B)$ for some $B \in \mathcal{B}^{X}$. Since by the hypothesis $X=\emptyset, \mathcal{B}^{X}=\{\emptyset\}$ follows, and $\mathcal{U}=\underline{P}(X \times X)$ by the definition of $\tau_{\mu}$. But $\mu \neq \emptyset$, and therefore $\mathcal{U} \supset \mathcal{V}$ for some $\mathcal{V} \in \mu$, showing that $\mathcal{U} \in \mu$ is true. Now let $X \neq \emptyset$. 
To (i) " $\geq$ ": Let $\mathcal{U} \in \mu$, hence, we can find $x \in X$, and $\{x\} \in \mathcal{B}^{X} \backslash\{\emptyset\}$ follows. Thus by definition $\mathcal{U} \in \tau_{\mu}(\{x\})$ is valid, and $\mathcal{U} \in \eta_{\tau_{\mu}}$ results.

" $\leq$ ": Conversely, $\mathcal{U} \in \eta_{\tau_{\mu}}$ implies $\mathcal{U} \in \tau_{\mu}(B)$ for some $B \in \mathcal{B}^{X}$. If $B \neq \emptyset$, then $\mathcal{U} \in \mu$ follows. If $B=\emptyset, \mathcal{U}=\underline{P}(X \times X)$ is valid, and $\mathcal{U} \in \mu$ results because $\mu \neq \emptyset$.

To (ii) $\geq$ : Without restriction let $B \in \mathcal{B}^{X} \backslash\{\emptyset\} . \mathcal{U} \in t(B)$ implies $\mathcal{U} \in \eta_{t}$, hence, $\mathcal{U} \in \tau_{\eta_{t}}(B)$ follows.

" $\leq$ ": $\mathcal{U} \in \tau_{\eta_{t}}(B)$ implies $\mathcal{U} \in \eta_{t}$. Then, there exists $D \in \mathcal{B}^{X}$ such that $\mathcal{U} \in t(D)$. If $D \neq \emptyset, \mathcal{U} \in t(B)$ follows. If $D=\emptyset, \mathcal{U}=\underline{P}(X \times X)$ holds and

$\dot{B} \times \dot{B} \in t(B)$, this implies $\mathcal{U} \in t(B)$ according To $\left(\mathrm{bc}_{1}\right)$. Evidently, the former established correspondence is functorial, meaning that for set-pointed equiform b-convergence spaces $\left(X, \mathcal{B}^{X}, \tau_{X}\right),\left(Y, \mathcal{B}^{Y}, \tau_{Y}\right)$ and every map $f: X \longrightarrow Y$ the following statements are equivalent:

(i) $f:\left(X, \mathcal{B}^{X}, \tau_{X}\right) \longrightarrow\left(Y, \mathcal{B}^{Y}, \tau_{Y}\right)$ is b-continuous;

(ii) $f:\left(X, \mathcal{B}^{X}, \eta_{\tau_{X}}\right) \longrightarrow\left(Y, \mathcal{B}^{Y}, \eta_{\tau_{Y}}\right)$ is b-uniformly continuous.

Remark 2.15. Now, we pointed out that SETeb-CONV can be even regarded as a bireflective subcategory of b-CONV.

Proof. For a b-convergence space $\left(X, \mathcal{B}^{X}, \tau\right)$, let us consider the b-convergence space $\left(X, \mathcal{B}^{X},\left(\left(\dot{\tau_{a}}\right)_{e}\right)\right)$, where $\tau_{a}, \tau_{e}$ are defined as in [7] and in general

$\dot{\tau}(\emptyset):=\{\underline{P}(X \times X)\}$ and

$\dot{\tau}(B):=\tau(B) \cup\left\{\mathcal{U} \in F I L(X \times X): \exists D \in \mathcal{B}^{X} \backslash\{\emptyset\}\right.$ such that $\left.\dot{D} \times \dot{D} \subset \mathcal{U}\right\}$ for each $B \in \mathcal{B}^{X} \backslash\{\emptyset\}$ and some b-convergence operator $\tau$.

It is easy to verify that $\left(X, \mathcal{B}^{X},\left(\left(\dot{\tau_{a}}\right)_{e}\right)\right)$ defines a set-pointed equiform b-convergence space such that

$$
1_{X}:\left(X, \mathcal{B}^{X}, \tau\right) \longrightarrow\left(X, \mathcal{B}^{X},\left(\left(\dot{\tau_{a}}\right)_{e}\right)\right)
$$

is b-continuous. Now, let $\left(Y, \mathcal{B}^{Y}, \tau_{Y}\right)$ be a set-pointed equiform b-convergence space and $f:\left(X, \mathcal{B}^{X}, \tau\right) \longrightarrow\left(Y, \mathcal{B}^{Y}, \tau_{Y}\right)$ be b-continuous map. We have to verify that $f:\left(X, \mathcal{B}^{X},\left(\left(\dot{\tau_{a}}\right)_{e}\right)\right) \longrightarrow\left(Y, \mathcal{B}^{Y}, \tau_{Y}\right)$ is b-continuous, too. For $B \in \mathcal{B}^{X} \backslash\{\emptyset\}$ let $\mathcal{U} \in\left(\left(\dot{\tau_{a}}\right)_{e}\right)(B)$. In case of $\mathcal{U} \in\left(\left(\dot{\tau_{a}}\right)_{e}\right)(B)$, we can find $x \in X$ such that $\mathcal{U} \in \tau_{a}(\{x\})$. Hence, by definition $\mathcal{U} \in \tau(D)$ for some $D \in \mathcal{B}^{X} \backslash\{\emptyset\}$ with $x \in D$. By the hypothesis we are getting $(f \times f)(\mathcal{U}) \in \tau_{Y}(f[D])=\tau_{Y}(f[B])$. Note also that $\left\{f[B]: B \in \mathcal{B}^{X}\right\} \subset \mathcal{B}^{Y}$ holds. If $\dot{D} \times \dot{D} \subset \mathcal{U}$ for some $D \in \mathcal{B}^{X} \backslash\{\emptyset\}$, we are getting $(f \times f)(\dot{D} \times \dot{D})=f[\dot{D}] \times f[\dot{D}] \in \tau_{Y}(f[D])=\tau_{Y}(f[B])$, and $(f \times f)(\mathcal{U}) \in \tau_{Y}(f[B])$ results, concluding the proof.

\section{On the Cartesian Closedness}

Cartesian closedness, i.e., the existence of natural function spaces is useful, e.g. in homotopy theory (fundamental groups) or for the constructing of completions [11]. In particular, it plays an important role in topological constructs. Moreover 
it should be noted that this property may be defined by means of a pair of adjoint functors $\left(\mathcal{F}_{1}, \mathcal{F}_{2}\right)$, where neither $\mathcal{F}_{1}$ nor $\mathcal{F}_{2}$ is an inclusion functor as in the preceding section dealing with reflections and coreflections.

Definition 3.1. A category $\underline{C}$ is called Cartesian closed provided that the following conditions are satisfied [11].

(1) For each pair $(X, Y)$ of $\underline{C}$-objects there exists a product $X \times Y$ in $\underline{C}$;

(2) For each $\underline{C}$-objects $X$, the following holds: for each $\underline{C}$-object $Y$ there exists some $\underline{C}$-object $Y^{X}$ (called power object) and some $\underline{C}$ morphism $e_{X, Y}: X \times Y^{X} \longrightarrow Y$ (called evaluation morphism) such that, for each $\underline{C}$-object $Z$ and each $\underline{C}$-morphism $f: X \times Z \longrightarrow Y$, there exists a unique $\underline{C}$-morphism $\widehat{f}: Z \longrightarrow Y^{X}$ such that the following diagram commutes.

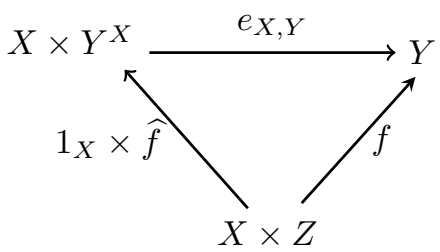

Remark 3.2. In topological constructs, the condition (1) is automatically fulfilled. Now it will be useful that in a topological construct $\underline{C}$ the property of being Cartesian closed is equivalent to the following statement: for each $\underline{C}$-object $Y$ and for any final epi-sink $\left(f_{i}: X_{i} \longrightarrow X\right)_{i \in I}$ in $\underline{C},\left(1_{Y} \times f_{i}: Y \times X_{i} \longrightarrow Y \times X\right)_{i \in I}$ is a final epi-sink, i.e., " $Y \times-$ " preserves final epi-sinks.

Theorem 3.3. Let $\left(f_{i}:\left(X_{i}, \mathcal{B}^{X_{i}}, \mu_{i}\right) \longrightarrow\left(X, \mathcal{B}^{X}, \mu\right)\right)_{i \in I}$ be an epi-sink in $\mathbf{b}-$ UFIL. Then, for any b-uniform filter space $\left(Y, \mathcal{B}^{Y}, \mu_{Y}\right),\left(1_{Y} \times f_{i}:\left(Y \times X_{i}, \mathcal{B}^{Y} \times\right.\right.$ $\left.\left.\mathcal{B}^{X_{i}}, \mu_{Y} \times \mu_{i}\right) \longrightarrow\left(Y \times X, \mathcal{B}^{Y} \times \mathcal{B}_{X}^{I}, \mu_{Y} \times \mu_{I}^{X}\right)\right)_{i \in I}$ is a final epi-sink in b-UFIL, see Proposition 2.2, Remark 2.9 and Proposition 2.11, respectively.

Proof. First let us consider the following diagram:

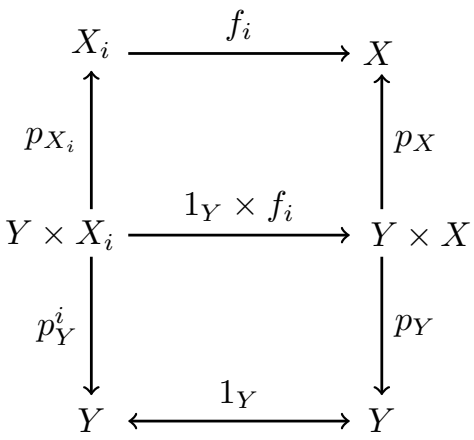

We will denote by $\left(\mathcal{B}^{Y} \times \mathcal{B}^{X_{i}}, \mu_{Y} \times \mu_{i}\right)$ and by $\left(\mathcal{B}^{Y} \times \mathcal{B}_{X}^{I}, \mu_{Y} \times \mu_{I}^{X}\right)$ the prevailing b-uniform filter product structures on its corresponding sets: 
Now we will show that $\left(1_{Y} \times f_{i}:\left(Y \times X_{i}, \mathcal{B}^{Y} \times \mathcal{B}^{X_{i}}, \mu_{Y} \times \mu_{i}\right) \longrightarrow\left(Y \times X, \mathcal{B}^{Y} \times\right.\right.$ $\left.\left.\mathcal{B}_{X}^{I}, \mu_{Y} \times \mu_{I}^{X}\right)\right)_{i \in I}$ is an epi-sink in b-UFIL.

If $\alpha, \beta:\left(Y \times X, \mathcal{B}^{Y} \times \mathcal{B}_{X}^{I}, \mu_{Y} \times \mu_{I}^{X}\right) \longrightarrow\left(Z, \mathcal{B}^{Z}, \mu_{Z}\right)$ are b-uniformly continuous maps such that $\alpha \circ\left(1_{Y} \times f_{i}\right)=\beta \circ\left(1_{Y} \times f_{i}\right)$ for each $i \in I$ and if $(y, x) \in Y \times X$ then since $X=\bigcup_{i \in I} f_{i}\left[X_{i}\right]$ (see Proposition 2.11), there is some $i \in I$ and some $x_{i} \in X_{i}$ with $f_{i}\left(x_{i}\right)=x$. Hence, $\alpha((y, x))=\alpha\left(\left(y, f_{i}\left(x_{i}\right)\right)\right)=\alpha\left(\left(1_{Y} \times f_{i}\right)\left(y, x_{i}\right)\right)=$ $\beta\left(\left(1_{Y} \times f_{i}\right)\left(\left(y, x_{i}\right)\right)\right)=\beta\left(\left(y, f_{i}\left(x_{i}\right)\right)=\beta((y, x))\right.$ and, consequently, $\alpha=\beta$ results.

Next, we will show that, for each $i \in I, 1_{Y} \times f_{i}:\left(Y \times X_{i}, \mathcal{B}^{Y} \times \mathcal{B}^{X_{i}}, \mu_{Y} \times \mu_{i}\right) \longrightarrow$ $\left(Y \times X, \mathcal{B}^{Y} \times \mathcal{B}_{X}^{I}, \mu_{Y} \times \mu_{I}^{X}\right)$ satisfies (buc 1$)$. So let for $i \in I, B_{i}^{*} \in \mathcal{B}^{Y} \times \mathcal{B}^{X_{i}}$. We have to verify that $\left(1_{Y} \times f_{i}\right)\left[B_{i}^{*}\right] \in \mathcal{B}^{Y} \times \mathcal{B}_{X}^{I}$ holds, which means $p_{Y}\left[\left(1_{Y} \times f_{i}\right)\left[B_{i}^{*}\right]\right] \in$ $\mathcal{B}^{Y}$ and $p_{X}\left[\left(1_{Y} \times f_{i}\right)\left[B_{i}^{*}\right]\right] \in \mathcal{B}_{X}^{I}$. By the hypothesis, we get $p_{Y}^{i}\left[B_{i}^{*}\right] \in \mathcal{B}^{Y}$ and $p_{X_{i}}\left[B_{i}^{*}\right] \in \mathcal{B}^{X_{i}}$. But $p_{Y}\left[\left(1_{Y} \times f_{i}\right)\left[B_{i}^{*}\right]\right] \subset p_{Y}^{i}\left[B_{i}^{*}\right]$ and $p_{X}\left[\left(1_{Y} \times f_{i}\right)\left[B_{i}^{*}\right] \subset f_{i}\left[p_{X_{i}}\left[B_{i}^{*}\right]\right]\right.$ hold since both parts of the diagram are commutative. Now the claim follows.

Next, the inclusion $\mathcal{B}^{Y} \times \mathcal{B}_{X}^{I} \subset \mathcal{B}_{Y \times X}^{I}$ is valid. $B^{*} \in \mathcal{B}^{Y} \times \mathcal{B}_{X}^{I}$ implies $p_{Y}\left[B^{*}\right] \in \mathcal{B}^{Y}$ and $p_{X}\left[B^{*}\right] \in \mathcal{B}_{X}^{I}$. Hence, there exists $i \in I$ and $B_{i} \in \mathcal{B}^{X_{i}}$ such that $p_{X}\left[B^{*}\right] \subset f_{i}\left[B_{i}\right]$. We set $B_{i}^{*}:=P_{Y}^{i}{ }^{-1}\left[p_{Y}\left[B^{*}\right]\right] \cap p_{X_{i}}^{-1}\left[B_{i}\right]$. Then, $B_{i}^{*} \in \mathcal{B}^{Y} \times \mathcal{B}^{X_{i}}$, since $p_{Y}^{i}\left[B_{i}^{*}\right]=p_{Y}^{i}\left[p_{Y}^{i}{ }^{-1}\left[p_{Y}\left[B^{*}\right] \cap p_{X_{i}}^{-1}\left[B_{i}\right]\right] \subset p_{Y}^{i}\left[p_{Y}^{i}{ }^{-1}\left[p_{Y}\left[B^{*}\right]\right]=p_{Y}\left[B^{*}\right] \in \mathcal{B}^{Y}\right.\right.$. $p_{X_{i}}\left[B_{i}^{*}\right]=p_{X_{i}}\left[p_{Y}\left[B^{*}\right] \cap p_{X_{i}}^{-1}\left[B_{i}\right]\right] \subset p_{X_{i}}\left[p_{X_{i}}^{-1}\left[B_{i}\right]\right]=B_{i} \in \mathcal{B}^{X_{i}}$. So it remains to verify that $B^{*} \subset\left(1_{Y} \times f_{i}\right)\left[B_{i}^{*}\right]$.

Let $(y, x) \in B^{*}$ imply $p_{X}(y, x)=x \in f_{i}\left[B_{i}\right]$. Hence, there exists $x_{i} \in B_{i}$ with $x=f_{i}\left(x_{i}\right)$. Now we put $z^{i}:=\left(y, x_{i}\right)$, consequently, $\left(1_{Y} \times f_{i}\right)\left(z^{i}\right)=\left(1_{Y} \times f_{i}\right)\left(y, x_{i}\right)$ implying $\left(y, f_{i}\left(x_{i}\right)\right)=(y, x)$. On the other hand, $z^{i} \in B_{i}^{*}$ is true, because $p_{Y}^{i}\left(z^{i}\right)=$ $p_{Y}^{i}\left(y, x_{i}\right)=y$ and $p_{X_{i}}\left(z^{i}\right)=p_{X_{i}}\left(y, x_{i}\right)=x_{i}$. Thus, our assumed inclusion holds. In addition $1_{Y} \times f_{i}:\left(Y \times X_{i}, \mathcal{B}^{Y} \times \mathcal{B}^{X_{i}}, \mu_{Y} \times \mu_{i}\right) \longrightarrow\left(Y \times X, \mathcal{B}^{Y} \times \mathcal{B}_{X}^{I}, \mu_{Y} \times \mu_{I}^{X}\right)$ satisfies $\left(\right.$ buc $\left._{2}\right)$ for each $i \in I$. So let for $i \in I, \mathcal{U}^{*} \in \mu_{Y} \times \mu_{i}$. We have to verify that $\left(\left(1_{Y} \times f_{i}\right) \times\left(1_{Y} \times f_{i}\right)\right)\left(\mathcal{U}^{*}\right) \in \mu_{Y} \times \mu_{I}^{X}$ is valid. By the hypothesis, we get $\left(p_{Y}^{i} \times p_{Y}^{i}\right)\left(\mathcal{U}^{*}\right) \in \mu_{Y}$ and $\left(p_{X_{i}} \times p_{X_{i}}\right)\left(\mathcal{U}^{*}\right) \in \mu_{i}$. Now we will show that

(i) $\left(p_{Y}^{i} \times p_{Y}^{i}\right)\left(\mathcal{U}^{*}\right) \subset\left(p_{Y} \times p_{Y}\right)\left(\left(1_{Y} \times f_{i}\right) \times\left(1_{Y} \times f_{i}\right)\right)\left(\mathcal{U}^{*}\right)$ and

(ii) $\left(f_{i} \times f_{i}\right)\left(\left(p_{X_{i}} \times p_{X_{i}}\right)\left(\mathcal{U}^{*}\right) \subset\left(p_{X} \times p_{X}\right)\left(\left(1_{Y} \times f_{i}\right) \times\left(1_{Y} \times f_{i}\right)\right)\left(\mathcal{U}^{*}\right)\right.$

are true. Then, the claim immediately follows.

To (i): $R^{*} \in\left(p_{Y}^{i} \times p_{Y}^{i}\right)\left(\mathcal{U}^{*}\right)$ implies $R^{*} \supset\left(p_{Y}^{i} \times p_{Y}^{i}\right)\left[U^{*}\right]$ for some $U^{*} \in \mathcal{U}^{*}$. It remains to prove the inclusion $\left(p_{Y}^{i} \times p_{Y}^{i}\right)\left[U^{*}\right] \supset\left(p_{Y} \times p_{Y}\right)\left[\left(\left(1_{Y} \times f_{i}\right) \times\left(1_{Y} \times f_{i}\right)\right)\left[U^{*}\right]\right]$.

$z \in\left(p_{Y} \times p_{Y}\right)\left[\left(\left(1_{Y} \times f_{i}\right) \times\left(1_{Y} \times f_{i}\right)\right)\left[U^{*}\right]\right]$ implies $z=\left(p_{Y} \times p_{Y}\right)(s)$ for some $s \in$ $\left(\left(1_{Y} \times f_{i}\right) \times\left(1_{Y} \times f_{i}\right)\right)\left[U^{*}\right]$, hence, $s=\left(\left(1_{Y} \times f_{i}\right) \times\left(1_{Y} \times f_{i}\right)\right)\left(u^{*}\right)$ for some $u^{*} \in U^{*}$. Consequently, $u^{*}=(u, v)$ for elements $u, v \in Y \times X_{i}$. It follows that $u=\left(y_{1}, x_{i}^{1}\right)$ and $v=\left(y_{2}, x_{i}^{2}\right)$ for $y_{1}, y_{2} \in Y$ and $x_{i}^{1}, x_{i}^{2} \in X_{i}$. Thus, we get $s=\left(\left(1_{Y} \times f_{i}\right) \times\left(1_{Y} \times\right.\right.$ $\left.\left.f_{i}\right)\right)\left(u^{*}\right)=\left(\left(1_{Y} \times f_{i}\right) \times\left(1_{Y} \times f_{i}\right)\right)(u, v)=\left(\left(1_{Y} \times f_{i}\right) \times\left(1_{Y} \times f_{i}\right)\right)\left(\left(y_{1}, x_{i}^{1}\right),\left(y_{2}, x_{i}^{2}\right)\right)=$ $\left.\left(\left(1_{Y} \times f_{i}\right)\left(y_{1}, x_{i}^{1}\right)\right),\left(1_{Y} \times f_{i}\right)\left(y_{2}, x_{i}^{2}\right)\right)=\left(\left(y_{1}, f_{i}\left(x_{i}^{1}\right)\right),\left(y_{2}, f_{i}\left(x_{i}^{2}\right)\right)\right.$. Hence,

$$
\begin{aligned}
z=\left(p_{Y} \times p_{Y}\right)(s) & =\left(p_{Y} \times p_{Y}\right)\left(\left(y_{1}, f_{i}\left(x_{i}^{1}\right)\right),\left(y_{2}, f_{i}\left(x_{i}^{2}\right)\right)\right. \\
& =\left(p _ { Y } \left(y_{1}, f_{i}\left(x_{i}^{1}\right), p_{Y}\left(y_{2}, f_{i}\left(x_{i}^{2}\right)\right)=\left(y_{1}, y_{2}\right) .\right.\right.
\end{aligned}
$$

On the other hand, we have $\left(p_{Y}^{i} \times p_{Y}^{i}\right)\left(u^{*}\right) \in\left(p_{Y}^{i} \times p_{Y}^{i}\right)\left[U^{*}\right]$ with $\left(p_{Y}^{i} \times p_{Y}^{i}\right)\left(u^{*}\right)=$ $\left(p_{Y}^{i} \times p_{Y}^{i}\right)((u, v))=\left(p_{Y}^{i}(u), p_{Y}^{i}(v)\right)=\left(p_{Y}^{i}\left(y_{1}, x_{i}^{1}\right), p_{Y}^{i}\left(y_{2}, x_{i}^{2}\right)=\left(y_{1}, y_{2}\right)\right.$.

To (ii): $R \in\left(f_{i} \times f_{i}\right)\left(\left(p_{X_{i}} \times p_{X_{i}}\right)\left(\mathcal{U}^{*}\right)\right)$ implies $R \supset\left(f_{i} \times f_{i}\right)[S]$ for some $S \in\left(p_{X_{i}} \times p_{X_{i}}\right)\left(\mathcal{U}^{*}\right)$. Consequently, $S \supset\left(p_{X_{i}} \times p_{X_{i}}\right)\left[U^{*}\right]$ for some $U^{*} \in \mathcal{U}^{*}$. 
Hence $\left.\left(f_{i} \times f_{i}\right)[S] \supset\left(f_{i} \times f_{i}\right)\left[\left(p_{X_{i}} \times p_{X_{i}}\right)\right]\left[U^{*}\right]\right]=\left(p_{X} \times p_{X}\right)\left[\left(\left(1_{Y} \times f_{i}\right) \times\left(1_{Y} \times\right.\right.\right.$ $\left.\left.\left.\left.f_{i}\right)\right)\left[U^{*}\right]\right] \in\left(p_{X} \times p_{X}\right)\left(\left(1_{Y} \times f_{i}\right) \times\left(1_{Y} \times f_{i}\right)\right)\left(\mathcal{U}^{*}\right)\right)$, because of the commutative diagram, and the claim follows.

Next, we will show that the inclusion $\mu_{Y} \times \mu_{I}^{X} \subset \mu_{I}^{Y \times X}$ holds.

$\mathcal{U}^{*} \in \mu_{Y} \times \mu_{I}^{X}$ implies $\left(p_{Y} \times p_{Y}\right)\left(\mathcal{U}^{*}\right) \in \mu_{Y}$ and $\left(p_{X} \times p_{X}\right)\left(\mathcal{U}^{*}\right) \in \mu_{I}^{X}$. Hence, we can find $i \in I$ and $\mathcal{U}_{i} \in \mu_{i}\left(f_{i} \times f_{i}\right)\left(\mathcal{U}_{i}\right) \subset\left(p_{X} \times p_{X}\right)\left(\mathcal{U}^{*}\right)$. Now we put : $\mathcal{U}_{i}^{*}:=\left\{R \subset\left(Y \times X_{i}\right) \times\left(Y \times X_{i}\right): \exists S \in\left(p_{Y} \times p_{Y}\right)\left(\mathcal{U}^{*}\right), \exists U \in \mathcal{U}_{i}\right.$ such that $R \supset$ $\left.\left(p_{Y}^{i} \times p_{Y}^{i}\right)^{-1}[S] \cap\left(p_{X_{i}} \times p_{X_{i}}\right)^{-1}[U]\right\}$. Then, $\mathcal{U}_{i}^{*} \in \mu_{Y} \times \mu_{i}$, since the following inclusions hold:

(1) $\left(p_{Y}^{i} \times p_{Y}^{i}\right)\left(\mathcal{U}_{i}^{*}\right) \supset\left(p_{Y} \times p_{Y}\right)\left(\mathcal{U}^{*}\right)$ and

(2) $\left(p_{X_{i}} \times p_{X_{i}}\right)\left(\mathcal{U}_{i}^{*}\right) \supset \mathcal{U}_{i}$.

To (1): $S \in\left(p_{Y} \times p_{Y}\right)\left(\mathcal{U}^{*}\right)$ implies $S \supset\left(p_{Y} \times p_{Y}\right)\left[U^{*}\right]$ for some $U^{*} \in \mathcal{U}^{*}$. Choose $U \in \mathcal{U}_{i}$, hence, $\left(p_{Y}^{i} \times p_{Y}^{i}\right)^{-1}\left[\left(p_{Y} \times p_{Y}\right)\left[U^{*}\right]\right] \cap\left(p_{X_{i}} \times p_{X_{i}}\right)^{-1}[U] \in \mathcal{U}_{i}^{*}$. Consequently, $\left(p_{Y}^{i} \times p_{Y}^{i}\right)\left[\left(p_{Y}^{i} \times p_{Y}^{i}\right)^{-1}\left[\left(p_{Y} \times p_{Y}\right)\left[U^{*}\right]\right] \in \mathcal{U}_{i}^{*}\right.$ follows, and $\left(p_{Y} \times p_{Y}\right)\left[U^{*}\right] \in \mathcal{U}_{i}^{*}$ results, showing that $S \in \mathcal{U}_{i}^{*}$ is valid.

To (2): For $U \in \mathcal{U}_{i}$, choose $U^{*} \in \mathcal{U}^{*}$, hence, $\left(p_{Y} \times p_{Y}\right)\left[U^{*}\right] \in\left(p_{Y} \times p_{Y}\right)\left(\mathcal{U}^{*}\right)$ and, consequently, $\left(p_{Y}^{i} \times p_{Y}^{i}\right)^{-1}\left[\left(p_{Y} \times p_{Y}\right)\left[U^{*}\right]\right] \cap\left(p_{X_{i}} \times p_{X_{i}}\right)^{-1}[U] \in \mathcal{U}_{i}^{*}$. But then $U=\left(p_{X_{i}} \times p_{X_{i}}\right)\left[\left(p_{X_{i}} \times p_{X_{i}}\right)^{-1}[U]\right] \in\left(p_{X_{i}} \times p_{X_{i}}\right)\left(\mathcal{U}_{i}^{*}\right)$ follows.

Now if we can show that $\left(\left(1_{Y} \times f_{i}\right) \times\left(1_{Y} \times f_{i}\right)\right)\left(\mathcal{U}_{i}^{*}\right) \subset \mathcal{U}^{*}$ is true, the proposed claim follows. $V \in\left(\left(1_{Y} \times f_{i}\right) \times\left(1_{Y} \times f_{i}\right)\right)\left(\mathcal{U}_{i}^{*}\right)$ implies $V \supset\left(\left(1_{Y} \times f_{i}\right) \times\left(1_{Y} \times f_{i}\right)\right)[R]$ for some $R \in \mathcal{U}_{i}^{*}$. Hence, we can find $S \in\left(p_{Y} \times p_{Y}\right)\left(\mathcal{U}^{*}\right)$ and $U \in \mathcal{U}_{i}$ such that $R \supset\left(p_{Y}^{i} \times p_{Y}^{i}\right)^{-1}[S] \cap\left(p_{X_{i}} \times p_{X_{i}}\right)^{-1}[U]$. Then, $\left(f_{i} \times f_{i}\right)[U] \in\left(p_{X} \times p_{X}\right)\left(\mathcal{U}^{*}\right)$ is valid according To $(2)$ and, consequently, $\left(f_{i} \times f_{i}\right)[U] \supset\left(p_{X} \times p_{X}\right)\left[U^{*}\right]$ for some $U^{*} \in \mathcal{U}^{*} . S \supset\left(p_{Y} \times p_{Y}\right)\left[V^{*}\right]$ for some $V^{*} \in \mathcal{U}^{*}$ and, thus, $S^{*}:=U^{*} \cap V^{*} \in \mathcal{U}^{*}$ follows. Now, it remains to verify that the inclusion $S^{*} \subset\left(\left(1_{Y} \times f_{i}\right) \times\left(1_{Y} \times f_{i}\right)\right)[R]$ holds. $z \in S^{*}$ implies $z \in U^{*} \cap V^{*}$, hence, $z=(r, v)$ for $r, v \in(Y \times X) \times(Y \times X)$ so that $r=\left(y_{1}, x_{1}\right)$ and $v=\left(y_{2}, x_{2}\right)$ for elements $y_{1}, y_{2} \in Y$ and $x_{1}, x_{2} \in X$. Hence, $\left(p_{X} \times p_{X}\right)(z)=\left(p_{X} \times p_{X}\right)(r, v)=\left(p_{X}(r), p_{X}(v)\right)=\left(p_{X}\left(y_{1}, x_{1}\right), p_{X}\left(y_{2}, x_{2}\right)\right)=$ $\left(x_{1}, x_{2}\right) \in\left(f_{i} \times f_{i}\right)[U]$ follows. But then we can find $w \in U$ such that $\left(x_{1}, x_{2}\right)=$ $\left(f_{i} \times f_{i}\right)(w)$ for $w=\left(z_{i}^{1}, z_{i}^{2}\right)$ with $z_{i}^{1}, z_{i}^{2} \in X_{i} \times X_{i} .\left(f_{i} \times f_{i}\right)(w)=\left(f_{i} \times f_{i}\right)\left(z_{i}^{1}, z_{i}^{2}\right)=$ $\left(f_{i}\left(z_{i}^{1}\right), f_{i}\left(z_{i}^{2}\right)\right)$, and $x_{1}=f_{i}\left(z_{i}^{1}\right), x_{2}=f_{i}\left(z_{i}^{2}\right)$ are resulting. Consequently, $\left(p_{Y} \times\right.$ $\left.p_{Y}\right)(z)=\left(p_{Y}(r), p_{Y}(v)\right)=\left(p_{Y}\left(y_{1}, x_{1}\right), p_{Y}\left(y_{2}, x_{2}\right)\right)=\left(y_{1}, y_{2}\right) \in S$ follows. On the other hand, $\left(\left(y_{1}, z_{i}^{1}\right),\left(y_{2}, z_{i}^{2}\right)\right) \in\left(p_{Y}^{i} \times p_{Y}^{i}\right)^{-1}[S] \cap\left(p_{X_{i}} \times p_{X_{i}}\right)^{-1}[U]$ is true because $\left(p_{Y}^{i} \times p_{Y}^{i}\right)\left(\left(y_{1}, z_{i}^{1}\right),\left(y_{2}, z_{i}^{2}\right)\right)=\left(p_{Y}^{i}\left(y_{1}, z_{i}^{1}\right), p_{Y}^{i}\left(y_{2}, z_{i}^{2}\right)\right)=\left(y_{1}, y_{2}\right) \in S$ by the hypothesis, since $\left(p_{X_{i}} \times p_{X_{i}}\right)\left(\left(y_{1}, z_{i}^{1}\right),\left(y_{2}, z_{i}^{2}\right)\right)=\left(p_{X_{i}}\left(y_{1}, z_{i}^{1}\right), p_{X_{i}}\left(y_{2}, z_{i}^{2}\right)\right)=$ $\left(z_{i}^{1}, z_{i}^{2}\right) \in U$ implying $\left(\left(y_{1}, z_{i}^{1}\right),\left(y_{2}, z_{i}^{2}\right)\right) \in R$ and, consequently, $\left(\left(1_{Y} \times f_{i}\right) \times\left(1_{Y} \times\right.\right.$ $\left.\left.f_{i}\right)\right)\left(\left(y_{1}, z_{i}^{1}\right),\left(y_{2}, z_{i}^{2}\right)\right) \in\left(\left(1_{Y} \times f_{i}\right) \times\left(1_{Y} \times f_{i}\right)\right)[R]$. But $z=\left(\left(1_{Y} \times f_{i}\right) \times\left(1_{Y} \times\right.\right.$ $\left.\left.f_{i}\right)\right)\left(\left(y_{1}, z_{i}^{1}\right),\left(y_{2}, z_{i}^{2}\right)\right)$. Taking our results into account, we get $\mathcal{B}^{Y} \times \mathcal{B}_{X}^{I} \subset \mathcal{B}_{Y \times X}^{I}$ and $\mu_{Y} \times \mu_{I}^{X} \subset \mu_{I}^{Y \times X}$, which means $\left(\mathcal{B}^{Y} \times \mathcal{B}_{X}^{I}, \mu_{Y} \times \mu_{I}^{X}\right) \leq\left(\mathcal{B}_{X \times Y}^{I}, \mu_{I}^{Y \times X}\right)$. But then the equality holds, since $\left(\mathcal{B}_{X \times Y}^{I}, \mu_{I}^{Y \times X}\right)$ is the finest b-uniform filter structure on $Y \times X$ with respect to the given data, see Proposition 2.11 .

Remark 3.4. Since b-UFIL is a Cartesian closed topological construct, it follows that quotient maps are finitely productive, but not necessarily productive (i.e., not closed under the formation of arbitrary products). Later, we will see that, in b-UFIL, this nice property holds in addition. 


\section{ON ONE-POINT EXTENSIONS}

As seen above, final epi-sinks play an important role if one considers Cartesian closedness. In what follows, they also are of interest in connection with the socalled extensionality of a topological construct $\underline{C}$ because, in $\underline{C}$, final epi-sinks are hereditary if and only if $\underline{C}$ is extensional, see [11].

Definition 4.1. A topological construct $\underline{C}$ is called extensional (hereditary) provided that every $\underline{C}$-object $X$ has a one-point extension $X^{*}$ as $\underline{C}$-object, i.e., every $\underline{C}$-object $X$ can be embedded via the addition of a single point $\infty$ into a

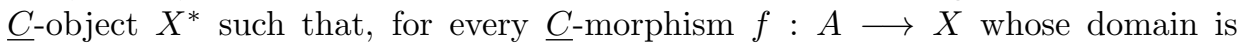
a subobject of an $\underline{C}$-object $Y$, the map $f^{*}: Y \longrightarrow X^{*}$ defined by

$$
f^{*}(y):=\left\{\begin{aligned}
f(y), & \text { if } \quad y \in A \\
\infty, & \text { if } \quad y \in Y \backslash A .
\end{aligned}\right.
$$

is a $\underline{C}$-morphism, i.e., the following diagram commutes.

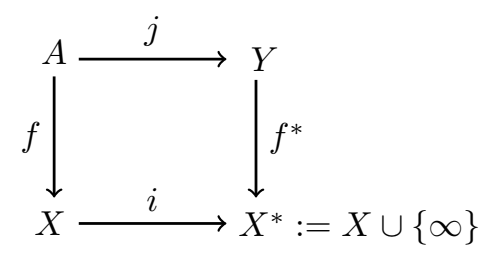

Theorem 4.2. In $\boldsymbol{b}$-UFIL every b-uniform filter space $\left(X, \mathcal{B}^{X}, \mu\right)$ has a onepoint extension.

Proof. For a b-uniform filter space $\left(X, \mathcal{B}^{X}, \mu\right)$, we put $X^{*}:=X \cup\{\infty\}$ with $\infty \notin X$. Now, we set $\mathcal{B}^{X^{*}}:=\mathcal{B}^{X} \cup\{\{\infty\}\}$ and $\mu^{*}:=\left\{\mathcal{V} \in F I L\left(X^{*} \times X^{*}\right)\right.$ : $\exists \mathcal{U} \in \mu$ such that $\left.\mathcal{V} \supset \mathcal{U}^{*}\right\}$, where $\mathcal{U}^{*}:=\left\{U^{*}: U \in \mathcal{U}\right\}$ with $U^{*}:=U \cup\left(X^{*} \times\right.$ $\{\infty\}) \cup\left(\{\infty\} \times X^{*}\right)$. Then, we claim that $\left(X^{*}, \mathcal{B}^{X^{*}}, \mu^{*}\right)$ is the one-point extension of $\left(X, \mathcal{B}^{X}, \mu\right)$ in b-UFIL. Evidently, $\left(\mathcal{B}^{X^{*}}, \mu^{*}\right)$ satisfies the axioms (buf 1$)$, $\left(\right.$ buf $\left._{2}\right)$ and $\left(\right.$ buf $\left._{4}\right)$ for being a b-uniform filter structure on $X^{*}$.

To (buf $)_{3}$ : Let $B \in \mathcal{B}^{X^{*}} \backslash\{\emptyset\}$. In the first case, if $B \in \mathcal{B}^{X} \backslash\{\emptyset\}$, then $\dot{B} \times \dot{B} \in \mu$ is valid. But $\dot{B} \times \dot{B} \supset(\dot{B} \times \dot{B})^{*}$ because $U^{*} \in(\dot{B} \times \dot{B})^{*}$ implies $U^{*}=U \cup\left(X^{*} \times\right.$ $\{\infty\}) \cup\left(\{\infty\} \times X^{*}\right)$ with $U \in \dot{B} \times \dot{B}$. Hence, $U \supset B \times B$, and $U^{*} \in \dot{B} \times \dot{B}$ follows. In the second case, if $B=\{\infty\}$, then choose $\mathcal{U} \in \mu$, hence, $\dot{B} \times \dot{B}=\dot{\infty} \times \dot{\infty} \supset \mathcal{U}^{*}$, since $U^{*} \in \mathcal{U}^{*}$ implies $U^{*}=U \cup\left(X^{*} \times\{\infty\}\right) \cup\left(\{\infty\} \times X^{*}\right)$ for some $U \in \mathcal{U}$. Consequently, $U^{*} \supset\{\infty\} \times\{\infty\}$, and $U^{*} \in \dot{B} \times \dot{B}$ is true.

Further, we indicate that $\left(X, \mathcal{B}^{X}, \mu\right)$ is a b-uniform filter subspace of

$$
\left(X^{*}, \mathcal{B}^{X^{*}}, \mu^{*}\right)
$$

which means that $\left(\mathcal{B}^{X}, \mu\right)$ is the coarsest b-uniform filter structure on $X$ such that $i:\left(X, \mathcal{B}^{X}, \mu\right) \longrightarrow\left(X^{*}, \mathcal{B}^{X^{*}}, \mu^{*}\right)$ is b-uniformly continuous. Evidently, the inclusion map $i: X \longrightarrow X^{*}$ is buc. Now, let $\left(\mathcal{A}^{X}, \mu_{X}\right)$ be a b-uniform filter structure on $X$ such that $i:\left(X, \mathcal{A}^{X}, \mu_{X}\right) \longrightarrow\left(X^{*}, \mathcal{B}^{X}, \mu^{*}\right)$ is buc, hence, $\mathcal{A}^{X} \subset$ 
$\mathcal{B}^{X}$. Since $A \in \mathcal{A}^{X}$ implies $i(A)=A \neq\{\infty\}, A \in \mathcal{B}^{X}$ follows. Now let $\mathcal{V} \in$ $\mu_{X}$, then, by the hypothesis, $(i \times i)(\mathcal{V}) \in \mu^{*}$. At once we can find $\mathcal{U} \in \mu$ with $(i \times i)(\mathcal{V}) \supset \mathcal{U}^{*}$. Our goal is to verify $\mathcal{U} \subset \mathcal{V} . \quad U \in \mathcal{U}$ implies $U^{*} \in(i \times i)(\mathcal{V})$, hence, $U^{*} \supset(i \times i)[V]$ for some $V \in \mathcal{V}$. But $(i \times i)[V]=V$, and $\left(x_{1}, x_{2}\right) \in V$ implies $x_{1} \neq \infty \neq x_{2}$. Consequently, $U \supset V$ follows, and $U \in \mathcal{V}$ results. Taking our two results into account, we get $\left(\mathcal{A}^{X}, \mu_{X}\right) \leq\left(\mathcal{B}^{X}, \mu\right)$, which proves the claim.

Next, let $\left(Y, \mathcal{B}^{Y}, \mu_{Y}\right)$ be a b-uniform filter space, $\left(A, \mathcal{B}^{A}, \mu_{A}\right)$ b-uniform filter subspace of $\left(Y, \mathcal{B}^{Y}, \mu_{Y}\right)$ and $f:\left(A, \mathcal{B}^{A}, \mu_{A}\right) \longrightarrow\left(X, \mathcal{B}^{X}, \mu\right)$ b-uniformly continuous map, then we claim that $f^{*}:\left(Y, \mathcal{B}^{Y}, \mu_{Y}\right) \longrightarrow\left(X^{*}, \mathcal{B}^{X^{*}}, \mu^{*}\right)$ is buc, where $f^{*}$ is defined by setting:

$$
f^{*}(y):=\left\{\begin{array}{rll}
f(y) & \text { for each } \quad y \in A \\
\infty & \text { for each } \quad y \in Y \backslash A
\end{array}\right.
$$

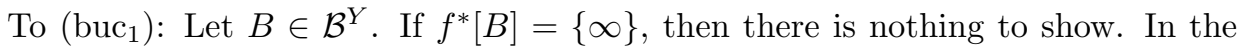
case of $\infty \notin f^{*}[B], B \cap A \in \mathcal{B}^{A}$, and, by the hypothesis, $f[B \cap A] \in \mathcal{B}^{X}$ follows. We will show that $f^{*}[B] \subset f[B \cap A]$ holds. $z \in f^{*}[B]$ implies $z=f^{*}(y)$ for some $y \in B$. If assuming $y \in Y \backslash A, f^{*}(y)=\infty$ follows, which is a contradiction. Hence, the claim is true.

To $\left(\right.$ buc $\left._{2}\right)$ : Let $\mathcal{U} \in \mu_{Y}$, hence, $\mathcal{U}_{A} \in \mu_{A}$, see Proposition 2.7. By the hypothesis, we get $(f \times f)\left(\mathcal{U}_{A}\right) \in \mu$. Now we will show that $\left(f^{*} \times f^{*}\right)(\mathcal{U}) \supset\left((f \times f)\left(\mathcal{U}_{A}\right)\right)^{*}$ is true. Let $R^{*}$ for $R \in(f \times f)\left(\mathcal{U}_{A}\right)$ be given, hence, $R \supset(f \times f)[V]$ for $V \in \mathcal{U}_{A}$. Then, $R^{*}=R \cup\left(X^{*} \times\{\infty\}\right) \cup\left(\{\infty\} \cup X^{*}\right) \supset(f \times f)(V)$ with $V=U \cap A \times A$ for some $U \in \mathcal{U}$ and, consequently, $\left(f^{*} \times f^{*}\right)[U] \in\left(f^{*} \times f^{*}\right)(\mathcal{U})$ is valid. It remains to verify $\left(f^{*} \times f^{*}\right)[U] \subset R^{*}$. Let $a \in\left(f^{*} \times f^{*}\right)[U]$, hence, $a=\left(f^{*} \times f^{*}\right)(b)$ for some $b \in U$, hence, $b=\left(y_{1}, y_{2}\right)$ for some pair $\left(y_{1}, y_{2}\right) \in U$. Consequently, $a=\left(f^{*}\left(y_{1}\right), f^{*}\left(y_{2}\right)\right)$ follows. In the case of $f^{*}\left(y_{1}\right)=\infty=f^{*}\left(y_{2}\right), a \in R^{*}$ results.

In the case of $\left(y_{1}, y_{2}\right) \in U \cap A \times A,\left(f^{*}\left(y_{1}\right), f^{*}\left(y_{2}\right)\right)=\left(f\left(y_{1}\right), f\left(y_{2}\right)\right)=(f \times$ $f)\left(y_{1}, y_{2}\right) \in(f \times f)[V] \subset R^{*}$, implying $a \in R^{*}$. If $f^{*}\left(y_{1}\right)=f\left(y_{1}\right)$ and $f^{*}\left(y_{2}\right)=\infty$, then $a=\left(f\left(y_{1}\right), \infty\right) \in X^{*} \times\{\infty\} \subset R^{*}$. In the case of $f^{*}\left(y_{1}\right)=\infty$ and $f^{*}\left(y_{2}\right)=$ $f\left(y_{2}\right), a=\left(\infty, f\left(y_{2}\right)\right) \in\{\infty\} \times X^{*} \subset R^{*}$.

Now, to sum up, we can state that b-UFIL forms a quasitopos as indicated.

\section{ON THE PRODUCTIVITY OF QUOTIENT MAPS}

In a topological construct $\underline{C}$ a $\underline{C}$-morphisms $f:(Y, \eta) \longrightarrow(X, \xi)$, where $(Y, \eta)$ and $(X, \xi)$ denote $\underline{C}$-objects is an extremal epimorphism in $\underline{C}$ if and only if $f$ is a quotient map, i.e., $f: X \longrightarrow Y$ is surjective, and $\xi$ is the final $\underline{C}$-structure on $X$, meaning that $(f: Y \longrightarrow X)$ is a final epi-sink. By transforming this fact into b-UFIL, we can state that $\xi$ consists of the pair $\left(\mathcal{B}_{X}^{f}, \mu_{f}^{X}\right)$ with $\mathcal{B}_{X}^{f}=\{B \subset$ $X: \exists D \in \mathcal{B}^{Y}$ such that $\left.f[D] \supset B\right\}$ and $\mu_{f}^{X}=\{\mathcal{U} \in F I L(X \times X): \exists \mathcal{V} \in$ $\mu_{Y}$ such that $\left.(f \times f)(\mathcal{V}) \subset \mathcal{U}\right\}$, where $\left(Y, \mathcal{B}^{Y}, \mu_{Y}\right)$ denotes the proposed b-uniform filter space. Then, in b-UFIL the following proposition holds:

Proposition 5.1. In b-UFIL, products of quotient maps are quotient maps again. 
Proof. In order to prove this statement in b-UFIL, let $\left(f_{i}:\left(Y_{i}, \mathcal{B}^{Y_{i}}, \mu_{Y_{i}}\right) \longrightarrow\right.$ $\left.\left(X_{i}, \mathcal{B}^{X_{i}}, \mu_{X_{i}}\right)\right)_{i \in I}$ be a non-empty family of quotient maps in b-UFIL and consider the following product diagram in b-UFIL, where $Y:=\prod_{i \in I} Y_{i}$ and $X=$ $\prod_{i \in I} X_{i}$, and $p_{Y_{i}}, p_{X_{i}}$, respectively, denote the prevailing projections, see Proposition 2.2 .

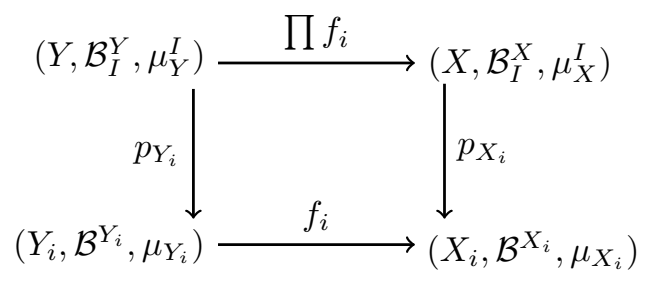

Since all $f_{i}$ are surjective, $\prod f_{i}$ is surjective. For each $i \in I, \mathcal{B}^{X_{i}}=\mathcal{B}_{X_{i}}^{f_{i}}$ and $\mu_{X_{i}}=\mu_{f_{i}}^{X_{i}}$, because $f_{i}$ is a quotient map. Now, we consider the pair $\left(\mathcal{E}^{X}, \mu_{X}\right)$, where $\mathcal{E}^{X}:=\left\{D \subset X: \exists G \in \mathcal{B}_{I}^{Y}\right.$ such that $\left.D \subset \prod f_{i}[G]\right\}$ and $\mu_{X}:=\{\mathcal{V} \in$ $F I L(X \times X): \exists \mathcal{W} \in \mu_{Y}^{I}$ such that $\left.\left(\prod f_{i} \times \prod f_{i}\right)(\mathcal{W}) \subset \mathcal{V}\right\}$. Then, the following equation holds: $\left(\mathcal{B}_{I}^{X}, \mu_{X}^{I}\right)=\left(\mathcal{E}^{X}, \mu_{X}\right)$, which indicates that $\prod f_{i}$ is a quotient map.

To " $\leq$ " : $B \in \mathcal{B}_{I}^{X}$ implies $p_{X_{i}}[B] \in \mathcal{B}^{X_{i}}$ for each $i \in I$. Thus, for each $i \in I$, there is some $B_{i} \in \mathcal{B}^{Y_{i}}$ with $p_{X_{i}}[B] \subset f_{i}\left[B_{i}\right]$. Then, $D:=\prod_{i \in I} B_{i} \in \mathcal{B}_{I}^{Y}$ with $B \subset$ $\prod f_{i}[D]$, because $x \in B$ implies $p_{X_{i}}(x)=x_{i} \in f_{i}\left[B_{i}\right]$. Hence, $x_{i}=f_{i}\left(z_{i}\right)$ for some $z_{i} \in B_{i}$ for each $i \in I$, and consequently $z=\left(z_{i}\right)_{i \in I} \in D$ with $\prod f_{i}(z)=z$ by using the commutativity of the product diagram. Consequently, $\mathcal{B}_{I}^{X} \subset \mathcal{E}^{X}$ is valid. Now let $\mathcal{U} \in \mu_{X}^{I}$, then, for each $i \in I,\left(p_{X_{i}} \times p_{X_{i}}\right)(\mathcal{U}) \in \mu_{X_{i}}$. Hence, we can find $\mathcal{U}_{i} \in \mu_{Y_{i}}$ with $\left(f_{i} \times f_{i}\right)\left(\mathcal{U}_{i}\right) \subset\left(p_{X_{i}} \times p_{X_{i}}\right)(\mathcal{U})$. If $j: \prod_{i \in I}\left(Y_{i} \times Y_{i}\right) \longrightarrow \prod_{i \in I} Y_{i} \times \prod_{i \in I} Y_{i}$ denotes the canonical isomorphism, i.e., $j\left(\left(y_{i}, z_{i}\right)\right)=\left(\left(y_{i}\right),\left(z_{i}\right)\right)$, and $\prod_{i \in I} \mathcal{U}_{i}$ the product filter on $\prod_{i \in I}\left(Y_{i} \times Y_{i}\right)$, then $j\left(\prod_{i \in I} \mathcal{U}_{i}\right)$ is a filter on $\prod_{i \in I} Y_{i} \times \prod_{i \in I} Y_{i}$ with $\left(p_{Y_{i}} \times p_{Y_{i}}\right)\left(j\left(\prod_{i \in I} \mathcal{U}_{i}\right)\right)=\mathcal{U}_{i}$ for each $i \in I$. Thus $j\left(\prod_{i \in I} \mathcal{U}_{i}\right) \in \mu_{Y}^{I}$. If $\widehat{j}: \prod\left(X_{i} \times X_{i}\right) \longrightarrow \prod_{i \in I} X_{i} \times \prod_{i \in I} X_{i}$ denotes the canonical isomorphism, then $\widehat{j}^{-1}\left(\prod f_{i} \times \prod f_{i}\right)\left(j\left(\prod_{i \in I} \mathcal{U}_{i}\right)\right) \subset \prod_{i \in I}\left(f_{i} \times f_{i}\right)\left(\mathcal{U}_{i}\right) \subset \prod_{i \in I}\left(p_{X_{i}} \times p_{X_{i}}\right)(\mathcal{U}) \subset \widehat{j}^{-1}(\mathcal{U})$. Hence, $\left(\prod f_{i} \times \prod f_{i}\right)\left(j\left(\prod_{i \in I} \mathcal{U}_{i}\right)\right) \subset \mathcal{U}$, which means that $\mathcal{U} \in \mu_{X}$. Thus, together we get $\left(\mathcal{B}_{I}^{X}, \mu_{X}^{I}\right) \leq\left(\mathcal{E}^{X}, \mu_{X}\right)$. Conversely, let $D \in \mathcal{E}^{X}$. Hence, we can find $G \in \mathcal{B}_{I}^{Y}$ with $D \subset \prod f_{i}[G]$. Then, $p_{Y_{i}}[G] \in \mathcal{B}^{Y_{i}}$ for each $i \in I$. Consequently, $f_{i}\left[p_{Y}[G]\right] \in \mathcal{B}_{X_{i}}^{f_{i}}$ follows for each $i \in I$. Since the diagram commutes, the equation $f_{i}\left[p_{Y}[G]\right]=p_{X_{i}}\left[\prod f_{i}[G]\right]$ holds for each $i \in I$. But the latter implies $\prod f_{i}[G] \in \mathcal{B}_{I}^{X}$, and $\mathcal{E}^{X} \subset \mathcal{B}_{I}^{X}$ follows. At last if $\mathcal{V} \in \mu_{X}$, then there exists $\mathcal{W} \in \mu_{Y}^{I}\left(\prod f_{i} \times\right.$ $\left.\prod f_{i}\right)(\mathcal{W}) \subset \mathcal{V}$. Furthermore, $\left(p_{X_{i}} \times p_{X_{i}}\right)\left(\left(\prod f_{i} \times \prod f_{i}\right)(\mathcal{W})\right)=\left(f_{i} \times f_{i}\right)\left(\left(p_{Y_{i}} \times\right.\right.$ $\left.\left.p_{Y_{i}}\right)(\mathcal{W})\right) \subset\left(p_{X_{i}} \times p_{X_{i}}\right)(\mathcal{V})$ for each $i \in I$. Consequently, $\left(p_{X_{i}} \times p_{X_{i}}\right)(\mathcal{V}) \in \mu_{X_{i}}$ is true for each $i \in I$, and $\mathcal{V} \in \mu_{X}^{I}$ results, which means that $\mu_{X} \subset \mu_{X}^{I}$ is valid and, thus, concludes the proof.

Remark 5.2. Following the terminology in [11], we can state that the category b-UFIL now forms a strong topological universe and, therefore, makes an important contribution to the convenient topology. 
Next, we will look at the behavior of certain subconstructs of b-UFIL, i.e., that of sb-UFIL, the full and isomorphism-closed subconstruct of b-UFIL whose objects are the symmetric b-uniform filter spaces and that of SETb-UFIL, the full and isomorphism-closed subconstruct of b-UFIL whose objects are the setconvergent b-uniform filter spaces, see [10].

\section{The topological Constructs sb-UFIL, B-FIL And SETB-UFIL}

First, let us recall the definitions for b-uniform filter spaces to be symmetric and setconvergent.

Definition 6.1. A b-uniform filter space $\left(X, \mathcal{B}^{X}, \mu\right)$ is called

(i) symmetric, provided that $\mu$ satisfies the following condition:

(s) $\mathcal{U} \in \mu$ implies $\mathcal{U}^{-1} \in \mu$, where $\mathcal{U}^{-1}:=\left\{R^{-1}: R \in \mathcal{U}\right\}$ with $R^{-1}:=$ $\{(x, z) \in X \times X:(z, x) \in R\}$

(ii) setconvergent, provided that $\left(\mathcal{B}^{X}, \mu\right)$ satisfies the following condition:

(sc) $\mathcal{U} \in \mu$ implies the existence of $B \in \mathcal{B}^{X} \backslash\{\emptyset\}$ and $\mathcal{F} \in F I L(X)$ such that $\dot{B} \times \mathcal{F} \in \mu$ with $\dot{B} \times \mathcal{F} \subset \mathcal{U}$.

By sb-UFIL and SETb-UFIL, we will denote the full subconstructs of b-UFIL, whose objects are the symmetric and setconvergent b-uniform filter spaces, respectively.

Remark 6.2. As seen in [10], sb-UFIL is bireflective as well as bicoreflective in b-UFIL and contains, in particular, the category of semiuniform convergence spaces in the sense of Preuß and the category b-FIL of b-filter spaces as bireflective and bicoreflective subconstruct in sb-UFIL. The last mentioned candidates can be regarded as spaces where the structures $\left(\mathcal{B}^{X}, \mu\right)$ are generated by all their $\mu$ Cauchy filters and, thus, play an important role if one considers the completeness of spaces.

In some special cases, filter merotopic spaces or filter spaces [5], respectively, can be recovered so that the Cauchy spaces or, more specifically, proximity spaces are integrated as well. In addition, SETb-UFIL is bicoreflective in b-UFIL, and it is isomorphic to the full subcategory RO-SETCONV of SETCONV, whose objects are the reordered set-convergence spaces. Let us mention that ROSETCONV is bireflective in SETCONV. Reordered set-convergence spaces are coming into play if one considers point-convergence on arbitrary $\underline{B}$-sets. In the classical case point-convergence on a set, $X$ can be regarded as a relation $q \subset$ $F I L(X) \times X$ satisfying certain conditions. Hence, the pair $(X, q)$ and the setconvergence space $\left(X, \mathcal{D}^{X}, \tau_{q}\right)$ are essentially the same. Here we note that $\mathcal{D}^{X}:=$ $\{\emptyset\} \cup\{\{x\}: x \in X\}$, and $\tau_{q}$ is defined by setting:

$\mathcal{F} \tau_{q} \emptyset$ if and only if $\mathcal{F}=\underline{P} X$

$B \in \mathcal{D}^{X} \backslash\{\emptyset\}$ implies $\mathcal{F} \tau_{q} B$ if and only if $\mathcal{F} q x$ for each $x \in B$.

Evidently $\left(X, \mathcal{D}^{X}, \tau_{q}\right)$ defines a reordered set-convergence space, which is repointed by satisfying the following more extended definition:

A set-convergence $\left(\mathcal{B}^{X}, \tau\right)$, where $\mathcal{B}^{X}$ is $\underline{B}$ - set and $\tau \subset F I L(X) \times \mathcal{B}^{X}$, is called repointed if $B \in \mathcal{B}^{X} \backslash\{\emptyset\}$ implies $\mathcal{F} \tau B$ if $\mathcal{F} \tau\{x\}$ for each $x \in B$. 
Evidently, each repointed set-convergence is reordered. But now, under this more general premise, we are able to consider point-convergence even on arbitrary $\underline{B}$ sets not only restricted to the discrete one. Thus, for example, point-convergence on the set of finite subsets, compact subsets or totally bounded subsets on a set $X$, respectively, can be now described and examined in addition to [4].

By the way, we also note that the full subconstruct rp-SETCONV of ROSETCONV, whose objects are the repointed set-convergence spaces is bireflective in RO-SETCONV. As essence we keep hold that b-UFIL is also a suitable candidate for studying point-convergence on a more general level than the classical one. Here we should note that the considered convergence in [4] can be equivalently described by certain discrete b-uniform filter structures and vice versa. In fact, let a convergence space $(X, \xi)$ be given in the sense of [4] then the space $\left(X, \mathcal{D}^{X}, \mu_{\xi}\right)$ , where $\mu_{\xi}:=\{\mathcal{U} \in F I L(X \times X): \exists \mathcal{F} \in F I L(X) \exists x \in X((\mathcal{F}, x) \in \xi$ and $\mathcal{U} \supset \mathcal{F} \times \dot{x}\}$ defines a specific discrete b-uniform filter space.

Conversely, if such a specific discrete b-uniform filter space $\left(X, \mathcal{D}^{X}, s\right)$ is given, then $\left(X, \eta_{s}\right)$ is a convergence space, where $(\mathcal{F}, x) \in \eta_{s}$ if and only if $\mathcal{F} \times \dot{x} \in s$. The just defined assignments are functorial and, thus, determine the proposed isomorphism.

Theorem 6.3. The topological construct sb-UFIL is Cartesian closed.

Proof. This statement can be proved by purely categorical arguments, see Remark 6.2. and [1].

Theorem 6.4. The topological construct b-FIL is Cartesian closed.

Proof. See the above stated references.

Theorem 6.5. The topological constructs $\mathbf{s b - U F I L ~ a n d ~} \mathbf{b}-\mathbf{F I L}$ are both hereditary.

Proof. Since b-UFIL is hereditary and sb-UFIL a bicoreflective subconstruct of b-UFIL, respectively b-FIL a bicoreflective subconstruct of sb-UFIL, which are both closed under formation of subspaces in its prevailing supercategories, hence, the statements made are true by applying purely categorical arguments.

Theorem 6.6. In the topological constructs $\mathbf{s b - U F I L ~ a n d ~ b - F I L , ~ t h e ~ p r o d u c t s ~}$ of quotient maps are quotient maps again.

Proof. Since sb-UFIL is bicoreflective in b-UFIL, closed under formation of products in b-UFIL and in b-UFIL quotients are productive, the claim follows by purely categorical arguments. Since b-FIL is bireflective in sb-UFIL closed under formation of quotient objects in sb-UFIL and in sb-UFIL quotients are productive, then, by using the above mentioned arguments, the claim results.

Corollary 6.7. The constructs sb-UFIL and b-FIL are forming strong topological universes. 


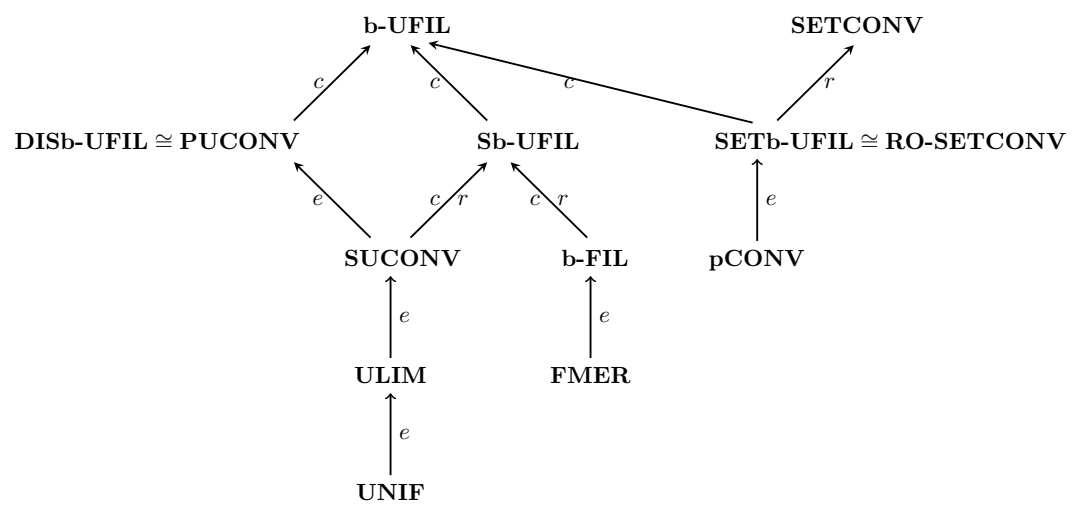

Legend:

$e:=$ embedding

$c:=$ bicoreflection

$r:=$ bireflection

$\cong:$ isomorphism

UFIL := Category of uniform limit spaces

UFIL:= Category of uniform spaces

FMER:= Category of filtermorotopic spaces

pCONV:= Categories of point-convergence spaces, e.g., KENT-convergence spaces, limit spaces, pseudotopological spaces, pretopological spaces, topological spaces, etc.

\section{REFERENCES}

[1] A. Behling, Einbettung uniformer Räume in topologische Universen, Dissertation, Freie Universität Berlin, Berlin, 1991.

[2] H. L. Bentley, H. Herrlich and E. Lowen-Colebunders, Convergence, Journal of Pure and Applied Algebra 68 (1990), 27-45.

[3] D. Doitchinov, A unified theory of topological, proximal and uniform spaces, Doklady Akademii Nauk SSSR 156 (1964), 21-24 (Russian), Soviet Mathematics Dokl. 5 (1964) 595-598 (English Translation).

[4] S. Dolecki and F. Mynard, Convergence Foundations of Topology, World Scientific, 2016.

[5] M. Katětov, On continuity structures and spaces of mappings, Commentationes Mathematicae Universitatis Carolinae 6 (1965), 275-278.

[6] D. C. Kent, Convergence functions and their related topologies, Fundamenta Mathematicae 54 (1964), 125-133.

[7] D. Leseberg, On topologically induced b-convergences, Topology Proceedings 37 (2011), 293-313.

[8] D. Leseberg, A new concept of convergence space, Mathematica Pannonica 19 (2008), 291303.

[9] D. Leseberg, Strict Topological Extensions and Power-Objects for B-Convergence, LAP Lambert Academic Publishing, Saarbrücken, 2015.

[10] D. Leseberg and Z. Vaziry, Categories of several convergences, Submitted to Journal of the American Mathematical Society.

[11] G. Preuß, Foundation of Topology, An Approach to Convenient Topology, Kluwer, 2002.

[12] A. Tozzi and O. Wyler, On categories of supertopological spaces. Acta Universitatis Carolinae. Mathematica et Physica 28 (1987), 137-149.

[13] O. Wyler, On convergence of filters and ultrafilters to subsets, in: H. Ehrig, H. Herrlich, H.-J. Kreowski and G. Preuß (eds.), Categorical Methods in Computer Science With Aspects from Topology, Lecture Notes in Computer Science 393, 1988, 340-350. 
Dieter Leseberg, Ernst Reuter Gesellschaft Berlin, Germany e-mail: leseberg@zedat.fu-berlin.de

Zohreh Vaziry, Department of Mathematics, Islamic Azad University, Karaj Branch, Karaj, Iran

e-mail: $\mathbf{z}_{-} \mathrm{m}_{-}$vaziry@yahoo.co.in 
